\title{
Asymptotic safety of gauge theories beyond marginal interactions
}

\section{Tugba Buyukbese* and Daniel F. Litim}

Department of Physics and Astronomy, U Sussex, Brighton, BN1 9QH, U.K.

E-mail: t.buyukbese@sussex.ac.uk, d.litimesussex.ac.uk

Following up on the recent disovery of asymptotic safety and exact interacting UV fixed points in four-dimensional gauge theories coupled to matter, we investigate the impact of higher dimensional operators using the method of functional renormalisation. In the Veneziano limit, we establish that classically irrelevant couplings take well-defined interacting fixed point values of their own, despite of their non-renormalisability within standard perturbation theory. We also establish vacuum stability in the presence of higher dimensional scalar operators. Universal scaling exponents are found as well, showing that the higher order couplings remain parametrically irrelevant with near-Gaussian values. Our results provide a crucial consistency check for exact asymptotic safety of weakly coupled gauge theories. Similarities with fixed points in other theories including $4 d$ quantum gravity are indicated.

34th annual International Symposium on Lattice Field Theory

24-30 July 2016

University of Southampton, UK

\footnotetext{
* Speaker.
} 


\section{Introduction}

Fixed points of the renormalisation group play an important role in quantum field theory. Infrared (IR) fixed points often control phase transitions and the spontaneous breaking of symmetry. Ultraviolet (UV) fixed points are key for a fundamental definition of the theory. A prominent example is asymptotic freedom where the UV fixed point is non-interacting [1,2]. UV fixed points can also be interacting, a scenario known as asymptotic safety [3,4]. Recently, it was discovered that four-dimensional gauge theories may develop exact UV fixed points [5, 6], including theorems of existence for weakly interacting fixed points in general gauge theories coupled to matter [7]. In general, finding interacting UV fixed points, however, offers novel challenges. For asymptotically free theories, anomalous dimensions vanish and the set of relevant, marginal, and irrelevant invariants is known beforehand. Provided a theory is asymptotically safe, anomalous dimensions are non-vanishing and residual interactions in the UV modify the power counting of invariants. In particular, the set of relevant and marginal invariants is then no longer known beforehand, and it cannot be taken for granted that invariants which are classically irrelevant will remain irrelevant at an interacting fixed point [8]. Moreover, higher order couplings may even fail to take fixed point values and thereby may spoil the interacting fixed point altogether.

In this note we investigate whether the recently discovered UV fixed points in four-dimensional gauge theories coupled to matter persists beyond the level of classically marginal couplings [5]. The challenge with this is that one has to deal with infinitely many new invariants. Moreover, higher order invariants have coupling constants with increasingly negative mass dimensions. One might hope that some progress can nevertheless be made provided the fixed point remains weakly coupled. To that end, we investigate the role of higher dimensional operators in the Veneziano limit, using the method of functional renormalisation which is based on a Wilsonian momentum cutoff [9]. The method gives access to powerlike as well as logarithmic divergences. It has been successfully applied to studies in $4 d$ quantum gravity [10] and in critical $3 d$ scalar theories $[11,12]$. Here, we apply the method to critical $4 d$ gauge theories [13].

\section{Asymptotic safety of gauge theories}

We begin by briefly recalling the basics of the asymptotically safe gauge-Yukawa model in four dimensions laid out in [5]. The theory contains $S U\left(N_{C}\right)$ gauge fields $A_{\mu}^{a}$ with field strength $F_{\mu \nu}^{a}\left(a=1, \cdots, N_{C}\right), N_{F}$ flavors of fermions $Q_{i}\left(i=1, \cdots, N_{F}\right)$ in the fundamental representation, and a $N_{F} \times N_{F}$ complex matrix scalar field $H$ uncharged under the gauge group. In the massless limit the action consists of the Yang-Mills action, the fermion kinetic terms, the Yukawa coupling, and the scalar kinetic and self-interaction $L=L_{\mathrm{YM}}+L_{\text {kin. }}+L_{\text {Yuk. }}+L_{\text {pot. }}$, where

$$
\begin{aligned}
L_{\mathrm{YM}} & =-\frac{1}{2} \operatorname{Tr} F^{\mu v} F_{\mu v} \\
L_{\text {kin. }} & =\operatorname{Tr}(\bar{Q} i \not D Q)+\operatorname{Tr}\left(\partial_{\mu} H^{\dagger} \partial^{\mu} H\right) \\
L_{\text {Yuk. }} & =-y \operatorname{Tr}(\bar{Q} H Q) \\
L_{\text {pot. }} & =-u \operatorname{Tr}\left(H^{\dagger} H H^{\dagger} H\right)-v\left(\operatorname{Tr} H^{\dagger} H\right)^{2} .
\end{aligned}
$$

The $\operatorname{Tr}$ denotes the trace over both color and flavor indices. The model has four classically marginal coupling constants given by the gauge coupling, the Yukawa coupling $y$, and the quartic scalar 
couplings $u$ and the "double-trace" scalar coupling $v$, which we write as

$$
\alpha_{g}=\frac{g^{2} N_{C}}{(4 \pi)^{2}}, \quad \alpha_{y}=\frac{y^{2} N_{C}}{(4 \pi)^{2}}, \quad \alpha_{h}=\frac{u N_{F}}{(4 \pi)^{2}}, \quad \alpha_{v}=\frac{v N_{F}^{2}}{(4 \pi)^{2}} .
$$

In order to achieve exact perturbative control we perform a Veneziano limit by sending both $N_{C}$ and $N_{F}$ to infinity while keeping their ratio $N_{F} / N_{C}$ constant. The latter becomes a freely tunable parameter and we are particularly interested in the regime where

$$
0 \leq \varepsilon \equiv \frac{N_{F}}{N_{C}}-\frac{11}{2} \ll 1
$$

which is a prerequisition for an asymptotically safe fixed point within the perturbative regime In terms of (2.3), and to the leading non-trivial order in perturbation theory, the running of couplings takes the form

$$
\begin{aligned}
& \beta_{g}=\frac{4}{3} \varepsilon \alpha_{g}^{2}+\left(25+\frac{26}{3} \varepsilon\right) \alpha_{g}^{3}-2\left(\frac{11}{2}+\varepsilon\right)^{2} \alpha_{g}^{2} \alpha_{y} \\
& \beta_{y}=(13+2 \varepsilon) \alpha_{y}^{2}-6 \alpha_{y} \alpha_{g} \\
& \beta_{h}=-(11+2 \varepsilon) \alpha_{y}^{2}+4 \alpha_{h}\left(\alpha_{y}+2 \alpha_{h}\right), \\
& \beta_{v}=12 \alpha_{h}^{2}+4 \alpha_{v}\left(\alpha_{v}+4 \alpha_{h}+\alpha_{y}\right) .
\end{aligned}
$$

To leading order in (2.3), the beta functions (2.4) display an interacting fixed point of order $\varepsilon$ in all classically marginal couplings,

$$
\alpha_{g}^{*}=\frac{26 \varepsilon}{57}, \quad \alpha_{y}^{*}=\frac{4 \varepsilon}{19}, \quad \alpha_{h}^{*}=\frac{\varepsilon}{19}(\sqrt{23}-1), \quad \alpha_{v}^{*}=-\frac{\varepsilon}{19}(2 \sqrt{23}-\sqrt{20+6 \sqrt{23}}) .
$$

We notice that the parametric smallness of the gauge coupling entails the smallness of the Yukawa and the quartic couplings. Moreover, vacuum stability of the theory is confirmed owing to $[5,6]$

$$
\alpha_{h}^{*}+\alpha_{v}^{*}=\frac{\varepsilon}{19}(\sqrt{20+6 \sqrt{23}}-\sqrt{23}-1)>0 .
$$

The UV fixed point has one UV relevant eigenvalue $\vartheta<0$ dominated by the gauge interactions

$$
\vartheta_{g}=-\frac{104}{171} \varepsilon^{2}, \quad \vartheta_{y}=\frac{52}{19} \varepsilon, \quad \vartheta_{1}=\frac{16}{19} \sqrt{23} \varepsilon, \quad \vartheta_{2}=\frac{8}{19} \sqrt{20+6 \sqrt{23}} \varepsilon .
$$

Consequently, the UV critical surface is one dimensional, and all other couplings are determined by the gauge coupling along the UV safe trajectories emmenating out of the fixed point.

\section{Results beyond classically marginal operators}

Next we address the impact of higher dimensional operators on the scalar sector. Various invariants can be formed out of complex square $N_{F} \times N_{F}$ matrices. Besides the invariants $\operatorname{Tr} H^{\dagger} H$ and $\operatorname{Tr}\left(H^{\dagger} H H^{\dagger} H\right)$ which already appear in the scalar potential (2.1) we may construct further invariants up to $N_{F}$ products $\operatorname{Tr}\left(H^{\dagger} H \cdots H^{\dagger} H\right)$, as well as powers and cross-products thereof. We are specifically interested in higher order invariants of the form

$$
V_{\text {higher }} \sim \sum_{n=2}^{\infty}\left(\lambda_{2 n-2}\left(\operatorname{Tr} H^{\dagger} H\right)^{n}+\lambda_{2 n-1} \operatorname{Tr}\left(H^{\dagger} H-\frac{1}{N} \operatorname{Tr} H^{\dagger} H\right)^{2} \cdot\left(\operatorname{Tr} H^{\dagger} H\right)^{n-1}\right) .
$$




\begin{tabular}{crccrc}
\hline coupling & fixed point & exponent & coupling & fixed point & exponent \\
\hline$\alpha_{3}^{*}$ & $0.442635 \varepsilon^{3}$ & $2+3.14773 \varepsilon$ & $\alpha_{4}^{*}$ & $0.197829 \varepsilon^{3}$ & $2+4.24573 \varepsilon$ \\
$\alpha_{5}^{*}$ & $-0.421820 \varepsilon^{4}$ & $4+4.19698 \varepsilon$ & $\alpha_{6}^{*}$ & $-0.0912196 \varepsilon^{4}$ & $4+5.29498 \varepsilon$ \\
$\alpha_{7}^{*}$ & $0.442354 \varepsilon^{5}$ & $6+5.24622 \varepsilon$ & $\alpha_{8}^{*}$ & $0.0561861 \varepsilon^{5}$ & $6+6.34422 \varepsilon$ \\
$\alpha_{9}^{*}$ & $-0.466105 \varepsilon^{6}$ & $8+6.29546 \varepsilon$ & $\alpha_{10}^{*}$ & $-0.0389432 \varepsilon^{6}$ & $8+7.39347 \varepsilon$ \\
$\alpha_{11}^{*}$ & $0.486798 \varepsilon^{7}$ & $10+7.34471 \varepsilon$ & $\alpha_{12}^{*}$ & $0.0287923 \varepsilon^{7}$ & $10+8.44271 \varepsilon$ \\
$\alpha_{13}^{*}$ & $-0.503072 \varepsilon^{8}$ & $12+8.39395 \varepsilon$ & $\alpha_{14}^{*}$ & $-0.0221745 \varepsilon^{8}$ & $12+9.49195 \varepsilon$ \\
\hline
\end{tabular}

Table 1: Fixed point values for the higher order scalar couplings $\alpha_{n}$ up to $n=14$ together with the universal scaling exponent to leading order in $\varepsilon$.

In (3.1) we have introduced the new couplings $\lambda_{n}$ in the scalar sector with canonical mass dimension $\left[\lambda_{n}\right]=2-n$ for even index $n \geq 4$ and $\left[\lambda_{n}\right]=1-n$ for odd index $n \geq 3$. Moreover, for any odd $n, \lambda_{n}$ and $\lambda_{n+1}$ have the same canonical mass dimension. We also introduce rescaled couplings

$$
\alpha_{n}=\lambda_{n}\left(\frac{N_{F}}{4 \pi}\right)^{c_{n}} \quad \text { with } \begin{cases}c_{n}=n & (n \text { even }) \\ c_{n}=n+1 & (n \text { odd })\end{cases}
$$

whereby perturbative loop factors and matter mulitplicities are scaled into the couplings, in line with our previous conventions (2.2). In order to find the RG equations for the new couplings $\alpha_{n}$ (3.2) we adopt the method of functional renormalisation, which is based on a Wilsonian version of the path integral where parts of the fluctuations have been integrated out [9]. The Wilsonian partition function takes the form

$$
Z_{k}[J]=\int D \varphi \exp \left(-S[\varphi]-\Delta S_{k}[\varphi]-\varphi \cdot J\right)
$$

where $S$ denotes the microscopic action, $\varphi$ stands for the various fields, and $J$ denotes an external current. The Wilsonian cutoff term at momentum scale $k$ is given explicitly as

$$
\Delta S_{k}[\varphi]=\frac{1}{2} \int \frac{d^{d} q}{(2 \pi)^{d}} \varphi(-q) R_{k}\left(q^{2}\right) \varphi(q) .
$$

The function $R_{k}$ obeys the limits $R_{k}\left(q^{2} \rightarrow 0\right)>0$ for $q^{2} / k^{2} \rightarrow 0$ and $R_{k}\left(q^{2}\right) \rightarrow 0$ for $k^{2} / q^{2} \rightarrow 0$ to guarantee that it acts as an IR momentum cutoff $[14,15]$. From (3.3) the "flowing" effective action $\Gamma_{k}$ follows via a Legendre transformation $\Gamma_{k}[\phi]=\sup _{J}\left(-\ln Z_{k}[J]+\phi \cdot J\right)+\Delta S_{k}[\phi]$, where $\phi=\langle\varphi\rangle_{J}$ denotes the expectation value of the quantum field. The renormalisation group scale-dependence of $\Gamma_{k}$ is given by

$$
\partial_{t} \Gamma_{k}=\frac{1}{2} \operatorname{Tr} \frac{1}{\Gamma_{k}^{(2)}+R_{k}} \partial_{t} R_{k} .
$$

It interpolates between the microscopic action $(k \rightarrow \infty)$ and the physical theory $(k \rightarrow 0)$ where all fluctuations are retained. The change with renormalisation group scale for the effective action $\Gamma_{k}$ is given in terms of an operator trace over the full propagator multiplied with the scale derivative of the cutoff itself. At weak coupling, iterative solutions of the flow (3.5) generate the conventional 


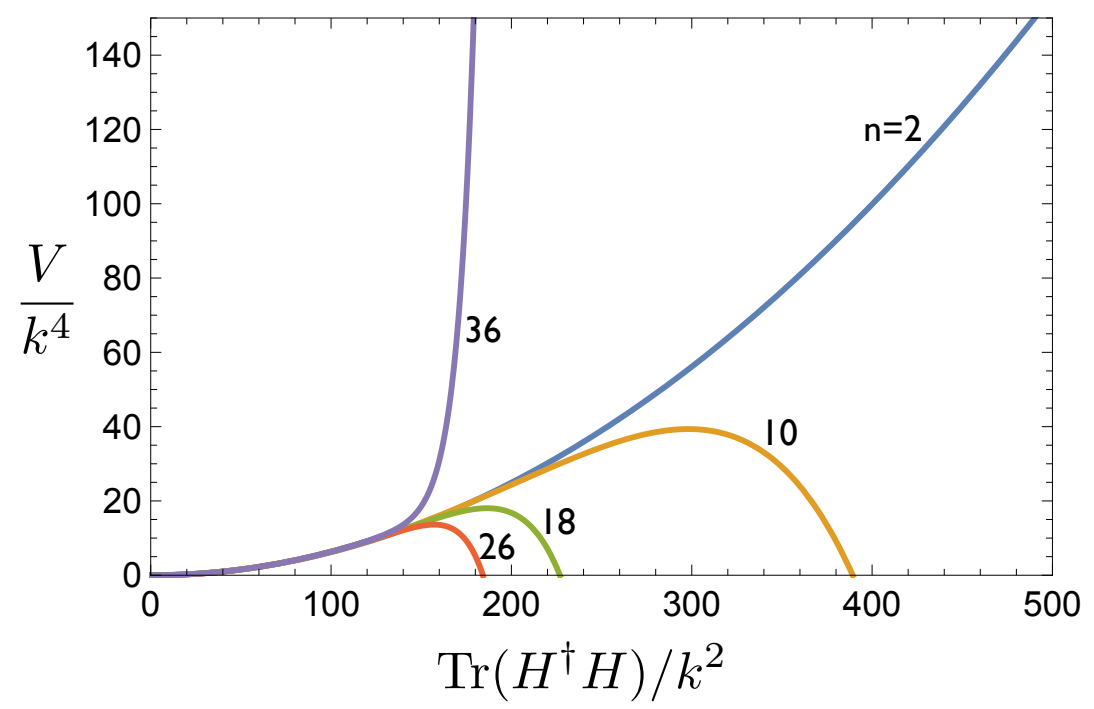

Figure 1: Dimensionless fixed point potential as a function of the dimensionless field in units of the RG scale $k$ including polynomial couplings $\alpha_{i}$ up to $i=n$, for $\varepsilon=0.01$ (see main text).

perturbative loop expansion [16]. Optimised choices for the regulator term [14, 15] allow for analytic flows and exact iterative solutions [11] even at strong coupling [12, 17]. Using the RG flow (3.5) for the model (2.1) and higher order scalar self interactions (3.1), we have analysed the corresponding beta functions for the flowing effective action and all polynomial couplings $\alpha_{n}$ [13]. We also confirm the beta functions (2.4) obtained within dimensional regularisation. Our fixed point results for the higher dimensional couplings are summarised in Tab. 1, and in Figs. 1 and 2. We have recursively solved the fixed point equations for all couplings $\alpha_{n}$, and to the leading non-trivial order in (2.3). As a result, all higher order couplings invariably take interacting fixed points, see Tab 1 . Moreover, we observe that higher order couplings are parametrically small, and increasingly so with increasing canonical mass dimension,

$$
\alpha_{n}^{*} \sim \varepsilon^{b_{n}} \quad \text { with } \quad b_{n}=2-\left[\lambda_{n}\right] / 2 .
$$

Notice that the power $b_{n}$ grows linearly with the canonical mass dimension of the coupling. For finite values of $\varepsilon$, the fixed points are found numerically. As soon as $\varepsilon$ takes finite values it is more convenient to obtain the couplings numerically. For $\varepsilon=0.01$ the results for the dimensionless scalar potential is shown in Fig. 1 as a $\frac{n+2}{2}$ th order polynomial of the dimensionless field $\operatorname{Tr}\left(H^{\dagger} H\right) / k^{2}$ for different values of $n$, also setting $\operatorname{Tr}\left(H^{\dagger} H-\frac{1}{N} \operatorname{Tr} H^{\dagger} H\right)^{2}=0$ in the plot. From Tab 1 we also observe that the couplings $\alpha_{2 i-1}^{*}$ and $\alpha_{2 i}^{*}$ arise with alternating signs leading to a radius of convergence of the order of $\sim 1 / \varepsilon$, see Fig. 1. For fields larger than the radius of convergence the polynomial approximation of the potential becomes obsolete and a numerical integration is performed [12, 13]. Our results establish vacuum stability in the presence of higher dimensional operators irrespective of the value for (2.3). The full effective potential at the UV fixed point (without polynomial approximation) comes out close to the $n=2$ curve provided $\varepsilon$ is sufficiently small. On the other hand, the impact of higher dimensional operators grows large with increasing $\varepsilon$. 


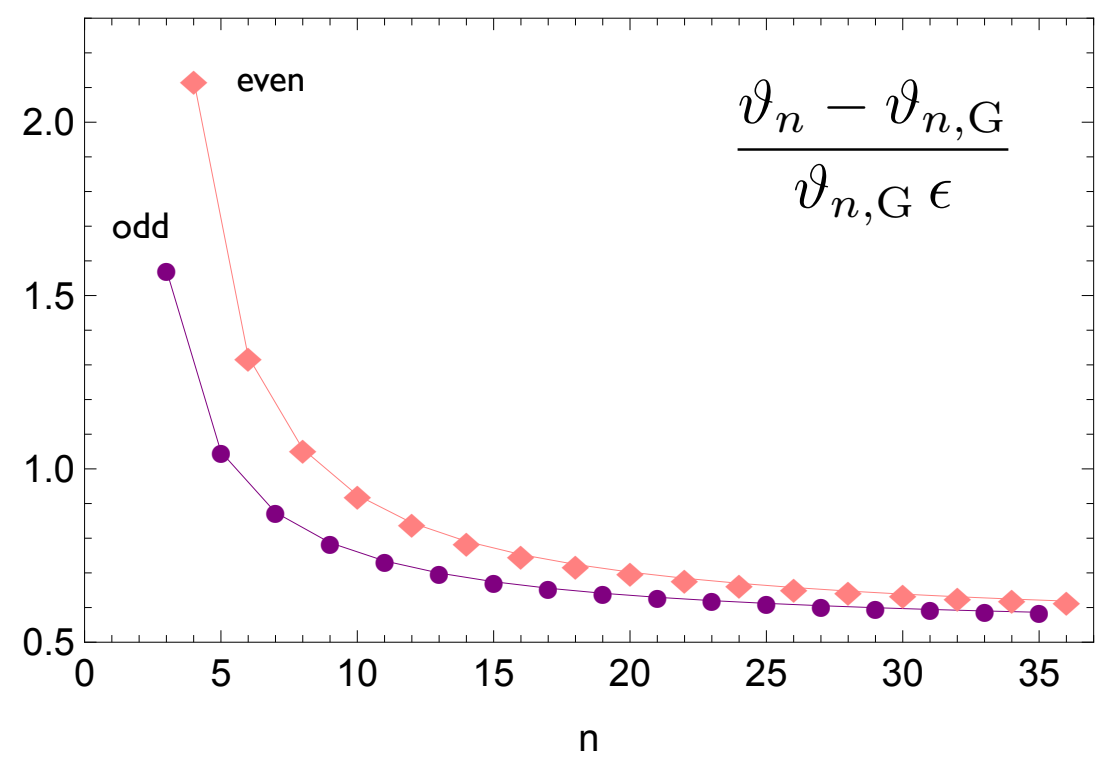

Figure 2: Shown is the fast approach to near-Gaussian scaling with increasing canonical mass dimension for all scaling exponents $\vartheta_{n}$ related to higher dimensional interactions $(\varepsilon=0.01)$.

We have also computed the set of universal scaling exponents $\left\{\vartheta_{n}\right\}$ complementing the result (2.7), and the first few of them are shown in Tab 1. We find that all higher dimensional operators remain irrelevant despite of interactions. The Gaussian scaling exponents are modified by parametrically small quantum corrections of the order of $\varepsilon$. As such, quantum corrections are not strong enough to turn irrelevant operators into relevant ones. Our findings are in accord with a recentlyproposed bootstrap search strategy for UV fixed points [8]. It was proposed to use the canonical mass dimension of couplings as an ordering principle [3], suggesting that the relevancy of invariants continues to be controlled by the canonical mass dimension even at an interacting fixed point [10]. Our results are an exact example for this type of behaviour: to see that even more explicitly, we show in Fig. 2 the relative deviation of scaling exponents from their Gaussian values,

$$
\frac{\vartheta_{n}-\vartheta_{n, G}}{\vartheta_{n, G}}=\Delta_{n} \cdot \varepsilon+\mathscr{O}\left(\varepsilon^{2}\right)
$$

with $\vartheta_{n, G}=-\left[\lambda_{n}\right]>0$ denoting the Gaussian exponents. A few comments are in order. Firstly, we observe that the relative variation (3.7) is of order $\varepsilon$, reflecting the fact that quantum corrections to scaling exponents are parametrically small in the regime (2.3). The coefficients $\Delta_{n}$ are shown in Fig. 2. We observe that $\Delta_{n}$ decrease with increasing $n$, further supporting the approach towards Gaussian scaling exponents. With increasing canonical mass dimension (and increasing $n$ ), we observe that the successive inclusion of two invariants with couplings $\left(\alpha_{2 n-1}, \alpha_{2 n}\right)$ and canonical mass dimension $2 n$ adds two scaling exponents to the eigenvalue spectrum which are both larger than all exponents from invariants with lower canonical mass dimension. This pattern is in complete agreement with the general bootstrap hypothesis put forward in [8]. 


\section{Conclusion}

We have established that the exact UV fixed point of [5] persists in the presence of higherdimensional interactions [13]. Our result offers an important consistency check by showing that higher dimensional couplings remain interacting, yet without spoiling the fixed point. Vacuum stability remains intact as well. It is intriguing that all higher order couplings take parametrically small fixed point values with universal scaling exponents close to Gaussian values. Hence, the smallness of (2.3) is also at the root for exact perturbative control for all higher dimensional invariants. It is interesting to note that similar results have been observed in asymptotically safe models of $4 d$ quantum gravity where a small underlying parameter is not available [10]. This strengthens the view that the "bootstrap hypothesis" of [8] is generally operative at interacting fixed points.

\section{References}

[1] D. J. Gross and F. Wilczek, Ultraviolet Behavior of Nonabelian Gauge Theories, Phys.Rev.Lett. 30 (1973) 1343-1346.

[2] H. D. Politzer, Reliable Perturbative Results for Strong Interactions?, Phys. Rev. Lett. 30 (1973) 1346-1349.

[3] S. Weinberg, Ultraviolet divergences in quantum theories of gravitation, . In General Relativity: An Einstein centenary survey, ed. S. W. Hawking and W. Israel, 790- 831.

[4] D. F. Litim, Renormalisation group and the Planck scale, Phil. Trans. Roy. Soc. Lond. A369 (2011) 2759-2778, [1102.4624].

[5] D. F. Litim and F. Sannino, Asymptotic safety guaranteed, JHEP 12 (2014) 178, [1 406 . 2337].

[6] D. F. Litim, M. Mojaza, and F. Sannino, Vacuum stability of asymptotically safe gauge-Yukawa theories, JHEP 01 (2016) 081, [1501.03061].

[7] A. D. Bond and D. F. Litim, Theorems for Asymptotic Safety of Gauge Theories, 1608.00519.

[8] K. Falls, D. Litim, K. Nikolakopoulos, and C. Rahmede, A bootstrap towards asymptotic safety, 1301.4191.

[9] C. Wetterich, Exact evolution equation for the effective potential, Phys.Lett. B301 (1993) 90-94.

[10] K. Falls, D. F. Litim, K. Nikolakopoulos, and C. Rahmede, Further evidence for asymptotic safety of quantum gravity, Phys. Rev. D93 (2016), no. 10 104022, [1410 . 4815].

[11] D. F. Litim and E. Marchais, Critical $O(N)$ models in the complex field plane, 1607.02030.

[12] A. Juttner, D. F. Litim, and E. Marchais, Global Wilson-Fisher fixed points, 1701.05168.

[13] T. Buyukbese and D. F. Litim, to appear, .

[14] D. F. Litim, Optimized renormalization group flows, Phys.Rev. D64 (2001) 105007 , [hep-th/0103195].

[15] D. F. Litim, Optimization of the exact renormalization group, Phys.Lett. B486 (2000) 92-99, [hep-th/0005245].

[16] D. F. Litim and J. M. Pawlowski, Perturbation theory and renormalization group equations, Phys.Rev. D65 (2002) 081701, [hep-th/ 0111191$].$

[17] D. U. Jungnickel and C. Wetterich, Effective action for the chiral quark-meson model, Phys. Rev. D53 (1996) 5142-5175, [hep-ph/9505267]. 